\title{
Оценка влияния исходных уравнений плотности и теплопроводности инея на результаты прогнозирования скорости формирования намо- роженного слоя
}

\author{
И. С. Козаченко ${ }^{\bowtie}$, А. Е.Лагутин \\ Одесская национальная академия пищевых технологий, ул. Канатная, 112, г. Одесса, 65039, Украина \\ $\triangle$ e-mail: ilya.s.kozachenko@gmail.com ORCID: https://orcid.org/0000-0002-5606-8640
}

\begin{abstract}
В статье приведены основные зависимости для определения плотности и теплопроводности инея. Приведено сравнение наиболее распространённых зависимостей теплопроводности и плотности инея. Представлень результаты расчета математической модели в виде графиков роста толщины слоя инея во времени на первом ряду, а также по глубине пучка. Проведен сравнительный анализ наиболее распространенных зависимостей плотности и теплопроводности инея и показано влияние этих параметров на итоговый вид функции зависимости толщины от времени. Проведена апробация модели на имеющихся экспериментальных данных для четырех режимов на различных температурных и влажностных уровнях. По итогу проведенной работы определена комбинация зависимостей плотности и теплопроводности инея, позволяющая обеспечить низкую погрешность математической модели для широкого диапазона расчетных режимов.
\end{abstract}

Ключевые слова: Моделирование; Воздухоохладитель; Формирование инея; Теплопроводность инея; Плотность инея; Тепло- и массообмен.

\section{Оцінка впливу вихідних рівнянь густини та теплопровідності інею на результати прогнозування швидкості формування намороженого шару}

I. С. Козаченко ${ }^{凶}$, А. Ю. Лагутін

Одеська національна академія харчових технологій, вул. Канатна, 112, м. Одеса, 65039, Україна

У статті наведені основні залежності для визначення густини та теплопровідності інею. Наведено порівняння найбільш поширених залежностей теплопровідності і густини інею. Представлені результати розрахунку математичної моделі у вигляді графіків зростання товщини шару інею в часі на першому ряду, а також по глибині пучка. Проведено порівняльний аналіз найбільш поширених залежностей густини і теплопровідності інею і показано вплив ции параметрів на підсумковий вид функиї залежності товщини від часу. Проведено апробацію моделі на наявних експериментальних даних для чотирьох режимів на різних температурних $і$ вологісних рівнях. За підсумком проведеної роботи визначено комбінація залежностей густини і теплопровідності інею, щзо дозволяє забезпечити низьку похибку математичної моделі для широкого діапазону розрахункових режимів.

Ключові слова: Моделювання; Повітроохолоджувач; Формування інею; Теплопровідність інею; Густина інею; Тепло- і масообмін.

(C) The Author(s) 2017. This article is an open access publication

This work is licensed under the Creative Commons Attribution 4.0 International License (CC BY) http://creativecommons.org/licenses/by/4.0/

\begin{tabular}{|llll|}
\hline & & \multicolumn{3}{c|}{ Условные обозначения } \\
$t$ & температура, ${ }^{\circ} \mathrm{C}$ & $\tau$ & время, час \\
$\varphi$ & относительная влажность, $\%$ & $G$ & объёмный расход, $\mathrm{M}^{3} / \mathrm{ч}$ \\
$h$ & высота, мм & $u$ & шаг, мм \\
$\delta$ & толщина, мм & $R e$ & число Рейнольдса \\
$D$ & диаметр, мм & $w$ & скорость воздуха, м/с \\
$d$ & влагосодержание, г/кг с.в. & $B$ & высота аппарата, м \\
$L$ & длина аппарата, м & $F, f$ & площадь, ${ }^{2}$ \\
$n$ & количество, штук & $\rho$ & плотность, кг $\mathrm{M}^{3}$ \\
$\lambda$ & коэффициент теплопроводности, Вт/м·К & $S$ & шаг труб, мм \\
$c_{1}, c_{2}$ & поправочные коэффициенты & & \\
\hline
\end{tabular}




\begin{tabular}{|llcl|}
\hline & & Индексы & \\
0 & параметры холодильного агента & $l$ & вход в ряд труб \\
2 & выход из ряда труб & $a$ & воздух \\
$f r$ & иней & $v r$ & вертикальный \\
$f$ & ребро & $i n$ & внутренний \\
$t b$ & труба & $e x$ & наружный \\
$b$ & основание & $h r$ & горизонтальный \\
$t p$ & вершина & $c$ & критический \\
$m$ & таяние & & \\
\hline
\end{tabular}

\section{1. Введение}

В области использования теплообменного оборудования для холодильных камер, явление образования инея на охлаждаемых поверхностях определяет качество работы и технические параметры аппаратов. Тем не менее, по настоящее время при проектировании воздухоохладителей оценка влияния инея осуществляется интуитивно. Так как иней радикально трансформирует базовый процесс переноса теплоты, его свойства и их влияние на работу воздухоохладителей должны быть подробно изучены. На данный момент существует множество зависимостей для определения плотности и теплопроводности инея как его основополагающих физических свойств, которые находят применение в математических моделях прогнозирования роста инея. В данной статье описывается влияние наиболее применяемых зависимостей на результаты расчета модели представленной авторами в предыдущей статье [21].

Теплофизические процессы, протекающие во внутренней структуре инея и влияющие на его рост, подчиняются действию сложных механизмов массопереноса а также их взаимодействию друг с другом. В своей работе Г.Н. Напалков [1] привел наиболее полный перечень подобных механизмов, в состав которых включил: молекулярную диффузию водяных паров, конвективное перемещение воздуха, излучение, теплопроводность составляющих внутренней структуры, фазовый переход водяных паров в твердое состояние, массоперенос взвешенных в воздухе мельчайших частиц льда под действием низкотемпературного градиента, перемещение под действием силы тяжести и закономерности роста кристаллов инея. В период формирования слоя инея, его толщина, плотность, теплопроводность и температура поверхности постепенно возрастают, а доля составляющих факторов в суммарном тепло- и массопереносе зависит от того, какую конфигурацию имеет холодная поверхность, какую ориентацию в пространстве она занимает, её температуры и интенсивности теплопереноса на границе раздела инейвоздух, т. е. от характера конвекции и параметров воздуха. Приведенный неполный перечень дает основание полагать, что теоретическое выведение зависимостей таких основополагающих свойств инея, как его теплопроводность и плотность, является трудоемкой задачей, попытки решения которой остаются актуальными по настоящее время. Тем не менее ряд авторов, имея достаточные объемы экспериментальных данных, для определения свойств инея [2-20,22] предлагают выведенные ими эмпирические зависимости. Как показал обзор существующих на сегодняшний день исследований в области определения плотности и теплопроводности инея, большинство авторов предлагают зависи- мости в диапазоне значений, которые либо выходит за рамки области значений применимых для камер охлаждения и хранения пищевых продуктов, либо покрывают её лишь частично.

Для точного моделирования процесса оседания инея необходимо полное понимание взаимосвязи между средней плотностью инея, его теплопроводностью и параметрами работы холодильной системы. Следовательно, уравнения, описывающие плотность и теплопроводность инея и заложенные в алгоритм расчета программы, являются основополагающими, а их влияние на результаты расчеты программы подлежит оценке.

\section{2. Плотность инея}

Несмотря на значимость плотности инея для математических моделей симуляции работы воздухоохладителей в доступной литературе встречается редко относительно выведенных зависимостей, подкрепленных надежной материальной базой и применяемых исследователями для моделирования процесса формирования инея. Еще меньше из них лежат вне области глубокого холода. Одними из первых в 1967 г. Hosoda Т. и Uzuhashi H. [15] представили уравнение зависимости плотности инея, как функцию температуры охлаждаемой поверхности и скорости потока воздуха. Полученное уравнение справедливо для температур поверхности $T_{f s}=-20 \ldots-5^{\circ} \mathrm{C}$, температуры омывающего воздуха $T_{1}=0 \ldots 10^{\circ} \mathrm{C}$, его скорости $w_{1}=1 \ldots 5 \mathrm{~m} / \mathrm{c}$ и влагосодержания $d_{1}=0,0188 \ldots 0,00604$ кг/кг с.в.

$$
\rho_{f r}=340 \cdot T_{f r}^{-0,445}+85 w_{1}
$$

Hayashi Y. [17] в 1977 г. предложил эмпирическую зависимость (2) основанную только на изменении температуры инея и применимую в области значений $T_{f r}=$ $-18,6 \ldots-5^{\circ} \mathrm{C}, w_{1}=2 \ldots 6 \mathrm{M} / \mathrm{c}, d_{1}>0,0075 \kappa \Gamma /$ кг с.в.

$$
\rho_{f r}=650 \exp \left[0,227\left(T_{f r}-273,15\right)\right] \text {. }
$$

Данная формула на сегодняшний день наиболее распространена за рубежом и часто применяется при моделировании прогнозирования роста инея. Тем не менее, в своем исследовании Hermes C. J. [18] определил, что уравнение Hayashi дает завышенные значения. Помимо этого, данная зависимость дает неприемлемо большие значения в диапазоне температур близких к точке таяния льда. На рисунке 1 представлено сравнение результатов Hermes, полученных в ходе проведения экспериментов с результатами вычисления плотности инея по формуле Hayashi для тех же условий. Анализ 
указывает на ограниченность применения данной формулы по причине сведения функции плотности инея лишь к температуре инея, что по мнению Hermes является слишком грубым упрощением. Несмотря на это, данное уравнение получило своё развитие. В 2012 г. группа ученых во главе с W. Wang [19] внесли в уравнение Hayashi доработки в виде дополнительных множителей (3).

$$
\rho_{f r}=650 \exp \left(0,227 T_{f r}\right) \cdot c_{1} \cdot c_{2},
$$

где:

$$
\begin{gathered}
\mathrm{C}_{1}=0,70132-0,11346 \mathrm{~T}_{\mathrm{rf}}-0,00203 \mathrm{~T}_{\mathrm{fr}}{ }^{2} \\
\mathrm{C}_{2}=1,4333-0,17389 \mathrm{~T}_{1}-0,00722 \mathrm{~T}_{\mathrm{fr}}{ }^{2} .
\end{gathered}
$$

Уравнение (3) применимо для области: $\quad T_{f r}=-$ $16 \ldots-8^{\circ} \mathrm{C}, T_{1}=11 \ldots 19^{\circ} \mathrm{C}, \varphi_{1}=0,4 \ldots 0,8, w_{1}=5 \mathrm{M} / \mathrm{c}$.

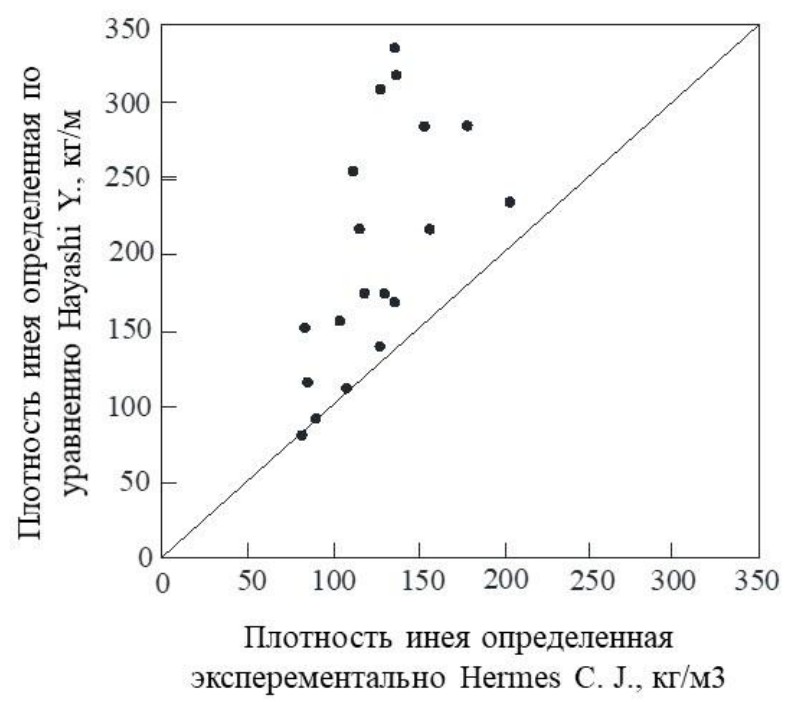

Рисунок 1 - График сравнения теоретических и экспериментальных значений плотности инея [18]

Н. В. Ломакиным [20] (1989 г.) было выведено уравнение (4) плотности инея. Как и в уравнении Hosoda и Uzuhashi плотность инея представлена в виде зависимости от скорости и температуры воздуха, температуры поверхности и толщины инея. По данным автора, уравнение проявляет хорошую сходимость с реально проведенными экспериментами

$$
\rho_{f r}=1562,5 \cdot\left(w_{1} \cdot \rho_{1}\right)^{0,15} \cdot \delta_{f r}^{0,35} \cdot \frac{T_{1}}{T_{f r}} .
$$

Hermes C. J. [18] в 2009 г. была представлена следующая зависимость (5):

$$
\rho_{f r}=a \cdot \exp \left(b \cdot T_{t b}+c \cdot T_{f r}\right),
$$

где константы $a, b, c$ были выведены на основании проведения экспериментов: $a=207,3 ; b=0,266 ; c=0,0615$.

Уравнение справедливо для области параметров: $T_{\mathrm{fr}}=-16 \ldots-4^{\circ} \mathrm{C}, T_{1}=16 \ldots 22^{\circ} \mathrm{C}, \varphi_{1}=0,5 \ldots 0,8, w_{1}=0,7 \mathrm{M} / \mathrm{c}$.

M. Kandula [12] в своей работе (2011 г.) приводит уравнение (7) для плотности инея как функции температуры поверхности инея, числа Рейнольдса, температуры охлаждаемой поверхности и температуры таянья льда. Здесь $\operatorname{Re}_{\mathrm{c}}=5 \cdot 10^{5}$ - критическое значения числа Рейнольдса для перехода от ламинарного режима к турбулентному, $T_{\mathrm{m}}$ - температура таянья льда

$$
\frac{\rho_{f r}}{\rho_{i c e}}=0,5 \frac{T_{f r}-T_{1}}{T_{t b}-T_{1}} \cdot \exp \left\{\begin{array}{c}
-\left[0,376+1,5\left(1-\frac{T_{f r}-T_{1}}{T_{t b}-T_{1}}\right)\right] \cdot \\
\cdot\left[1-\left(\frac{\mathrm{Re}}{\mathrm{Re}_{c}}\right)^{0,5}\right]
\end{array}\right\}
$$

Во всех вышеперечисленных случаях приведенные уравнения для плотности инея являются эмпирическими, что ограничивает их применимость в рамках экспериментов при которых они были выведены. Стоит отметить, что в основе большинства уравнений лежит температура поверхности инея, это значит, что их применение неразрывно связанно с изменением толщины инея во времени и не может быть применено к моделям отдельно от этих факторов.

\section{3. Теплопроводность инея}

Дать точное описание действительной теплопроводности инея - чрезвычайно сложная задача. Это является следствием неравномерной пористости строения, наличия фракций льда различной формы и размеров, наличия различного количества влаги в воздухе, проникающей вглубь инея, и её взаимодействия влияющей на тепло и массоперенос. Пористая структура инея с наличием пор различных форм и размеров отдаляют процесс переноса теплоты от изотропного. По этой причине многие модели принимают допущение об однородности структуры инея по толщине. В таблице 1 представлена часть доступных в свободных источниках зависимостей теплопроводности инея накопленных с первой половины прошлого века. Так как во всех без исключения зависимостях теплопроводность является функцией плотности, имеет смысл расположить кривые данных зависимостей на одном графике, как это показано на рисунке 2.

Теплопроводность инея зависит от нескольких параметров, таких как температура инея, температура охлаждаемой поверхности а также параметров воздуха, омывающего иней (температура $t_{1}$, скорость $w_{\mathrm{a}}$, относительная влажность $\varphi_{1}$ и прочие). Наиболее весомым фактором является плотность инея. Ниже приведены эмпирические зависимости для определения теплопроводности инея, пригодных к использованию при построении математических моделей прогнозирования динамики роста инея.

Точное описание закона изменения теплопроводности инея является трудной задачей. Причиной тому является его переменная структура, которая варьируется от ледяных иголок, образующих пористую структуру до гомогенного льда, и его состояние, которое находится в непрерывном процессе трансформации как во временном поле, так и по вектору глубины слоя. 


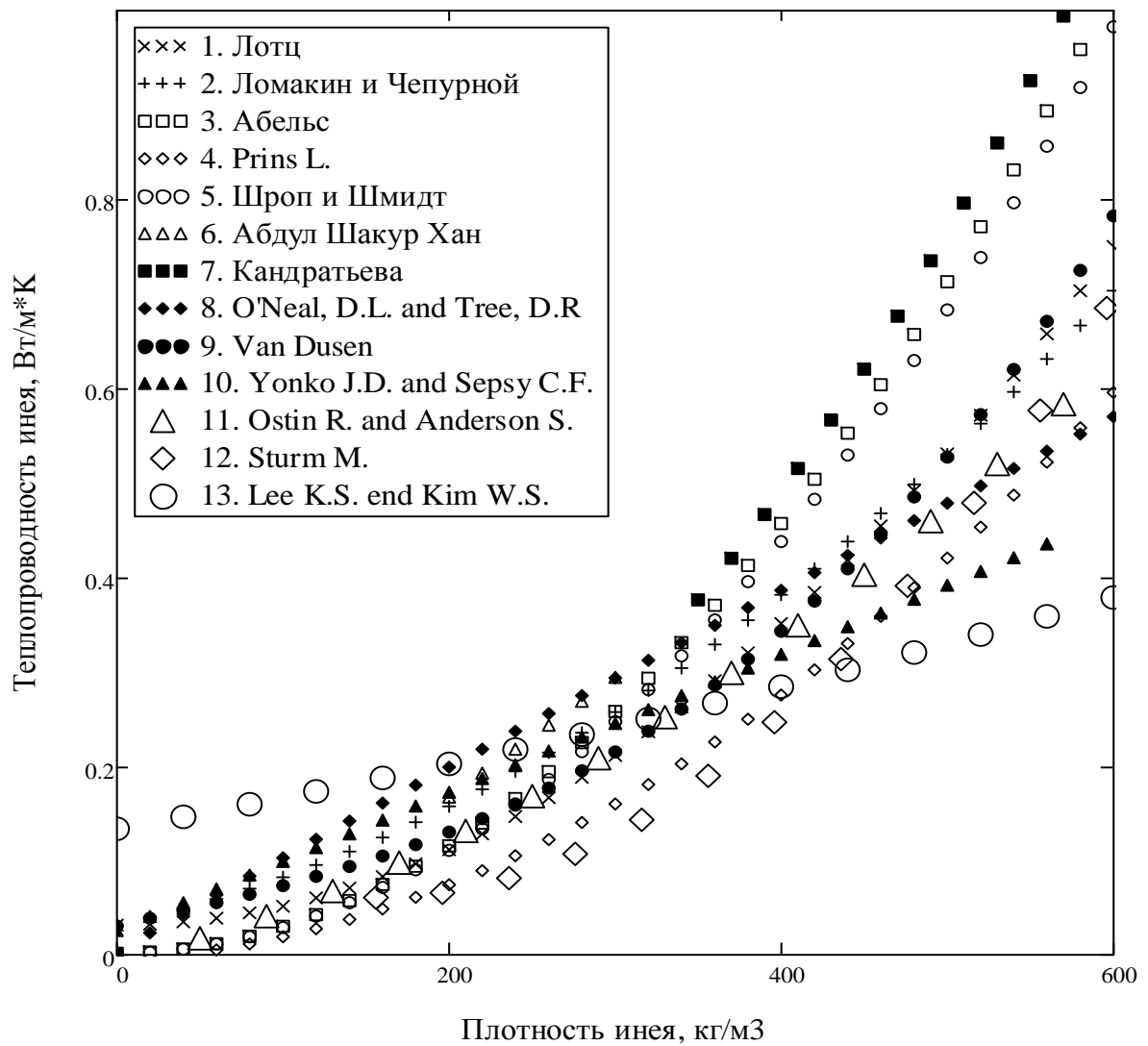

Рисунок 2 - График сравнения полученных различными авторами зависимостей теплопроводности инея от плотности инея

Tаблица 1 - Перечень зависимостей для определения теплопроводности инея

\begin{tabular}{|c|c|c|c|}
\hline $\begin{array}{c}\text { № } \\
\text { П.П. }\end{array}$ & Авторы & Зависимость & $\begin{array}{c}\text { Область примене- } \\
\text { ния }\end{array}$ \\
\hline 1 & Lotz H. [2] & $\lambda_{f r}=0,03+2 \cdot 10^{-6} \cdot \rho_{f r}^{2}$ & \\
\hline 2 & $\begin{array}{l}\text { Ломакин и Чепурной } \\
\text { [20] }\end{array}$ & $\lambda_{f r}=0,03\left(1+0,0064 \cdot \rho_{f r}^{2}\right)^{2}$ & \\
\hline 3 & Г. Ф. Абельс [1] & $\lambda_{f r}=2,85 \cdot 10^{-6} \cdot \rho_{f r}^{2}$ & \\
\hline 4 & Prins L. [4] & $\lambda_{f r}=1,5\left(\rho_{f r}^{2} \cdot 10^{-3}+0,0035\right)^{2}-0,01$ & \\
\hline 5 & Шроп и Шмидт [1] & $\lambda_{f r}=2,73 \cdot 10^{-6} \cdot \rho_{f r}^{2}$ & \\
\hline 6 & Абдул Шакур Хан [5] & $\lambda_{f r}=1,2676 \cdot 10^{-3} \cdot \rho_{f r}-0,0877$ & \\
\hline 7 & А. С. Кондратьева [1] & $\lambda_{f r}=3,06 \cdot 10^{-6} \cdot \rho_{f r}^{2}$ & $\rho_{\mathrm{fr}}>350 \kappa \Gamma / \mathrm{M}^{3}$ \\
\hline 8 & $\begin{array}{l}\text { O'Neal D.L. \& Tree D.R. } \\
\text { [7] }\end{array}$ & $\lambda_{f r}=1,202 \cdot 10^{-3} \cdot \rho_{f r}^{0,963}$ & $\rho_{\mathrm{fr}}<130 \mathrm{\kappa} / \mathrm{M}^{3}$ \\
\hline 9 & Van Dusen [8] & $\lambda_{f r}=0,029+0,403 \cdot 10^{-3} \cdot \rho_{f r}+0,2367 \cdot 10^{-8} \cdot \rho_{f r}^{3}$ & $243<\mathrm{t}_{\mathrm{fr}}<273^{\circ} \mathrm{K}$ \\
\hline 10 & $\begin{array}{l}\text { J. D. Yonko \& C. F. Sep- } \\
\text { sy [6] }\end{array}$ & $\lambda_{f r}=0,024248+0,731 \cdot 10^{-3} \cdot \rho_{f r}+0,1183 \cdot 10^{-5} \cdot \rho_{f r}$ & $\rho_{\mathrm{fr}}<573 \kappa \mathrm{\kappa} / \mathrm{M}^{3}$ \\
\hline 11 & $\begin{array}{l}\text { Ostin R. \& Anderson S. } \\
\text { [9] }\end{array}$ & $\lambda_{f r}=-8,71 \cdot 10^{-3}+4,39 \cdot 10^{-4} \cdot \rho_{f r}+1,05 \cdot 10^{-6} \cdot \rho_{f r}^{2}$ & $\begin{array}{l}50<\rho_{\mathrm{fr}}<680 \kappa \mathrm{\kappa г} / \mathrm{M}^{3} \\
253<\mathrm{t}_{\mathrm{fr}}<258^{\circ} \mathrm{K}\end{array}$ \\
\hline 12 & Sturm M. [10] & $\lambda_{f r}=0,138-1,01 \cdot 10^{-3} \cdot \rho_{f r}+3,233 \cdot 10^{-6} \cdot \rho_{f r}^{2}$ & $156<\rho_{\mathrm{fr}}<600 \kappa \Gamma / \mathrm{M}^{3}$ \\
\hline 13 & $\begin{array}{l}\text { Lee K.S. end Kim W.S. } \\
{[11]}\end{array}$ & $\lambda_{f r}=0,132+3,13 \cdot 10^{-4} \cdot \rho_{f r}+1,6 \cdot 10^{-7} \cdot \rho_{f r}^{2}$ & \\
\hline
\end{tabular}




\section{4. Результаты}

Практическое применение оценки функций зависимостей плотности и теплопроводности инея, используемых в модели прогнозирования интенсивности роста инея, состоит в построении зависимости $\delta_{f r}=f(\tau)$. Для оценки влияния данных параметров был проведен сравнительный расчет. Результаты представлены в виде графиков на рисунках 3-8. В математическую модель, в качестве исходных данных, были внесены параметры, полностью повторяющие данные из экспериментов, проведенных Бакулиным А. С. Геометрические параметры труб и пучков, для которых производился расчет, представлены в таблице 2. Режимы проведения эксперимента представлены в таблице 3. В качестве хладагента применялся аммиак. Воздухоохладитель это теплообменный пучок с шахматным расположением оребренных труб. Оребрение выполнено в виде круглого биметаллического ребра, нанесенного методом литья под давлением.

Tаблица 2 - Геометрические параметры труб и пучка

\begin{tabular}{|c|c|c|}
\hline № п.II. & Параметр & Значение \\
\hline 1 & Диаметр и толщина стенки трубы $\left(D_{\mathrm{tb}} \mathrm{x} \delta_{\mathrm{tb}}\right)$, мм & $25 \times 2,5$ \\
\hline 2 & Толщина наплавки ребра $\left(\delta_{\mathrm{fm}}\right)$, мм & 1,5 \\
\hline 3 & Диаметр ребра $\left(D_{\mathrm{f}}\right)$, мм & 80 \\
\hline 4 & Диаметр основания ребра $\left(D_{\mathrm{b} . \mathrm{f}}\right)$, мм & 28 \\
\hline 5 & Высота ребра $\left(h_{\mathrm{f}}\right)$, мм & 26 \\
\hline 6 & Толщина основания ребра $\left(\delta_{\text {b.f. }}\right)$, мм & 1,2 \\
\hline 7 & Толщина вершины ребра $\left(\delta_{\text {tp.f. }}\right)$, мм & 0,6 \\
\hline 8 & Поперечный шаг труб $\left(S_{\text {tb.vr. }}\right)$, мм & 120 \\
\hline 9 & Продольный шаг труб $\left(S_{\text {tb.hr. }}\right)$, мм & 90 \\
\hline 10 & Шаг ребра $\left(u_{\mathrm{f}}\right)$, мм & 20 \\
\hline 11 & Количество труб пучка по фронту пучка $\left(n_{\mathrm{vr}}\right)$, шт. & 8 \\
\hline 12 & Количество труб пучка по глубине пучка $\left(n_{\mathrm{hr}}\right)$, шт. & 8 \\
\hline
\end{tabular}

Таблица 3 - Режимы проведения эксперимента

\begin{tabular}{|l|c|c|c|c|c|c|}
\hline № п.п. & Режим, № по [22] & $\mathbf{t}_{\mathbf{1}},{ }^{\circ} \mathbf{C}$ & $\mathbf{t}_{\mathbf{0}},{ }^{\circ} \mathbf{C}$ & $\mathbf{G}_{\mathbf{a}}, \mathbf{M}^{\mathbf{3}} / \mathbf{4}$ & $\boldsymbol{\varphi}_{\mathbf{1}}, \boldsymbol{\%}$ & Область наблюдения \\
\hline 1 & 42 & 0 & -12 & 7272 & 85 & первый ряд \\
\hline 2 & 44 & -5 & -14 & 7272 & 90 & первый ряд \\
\hline 3 & 19 & 0 & -10 & 10800 & 95 & по глубине пучка \\
\hline 4 & 39 & -4 & -14 & 10800 & 95 & по глубине пучка \\
\hline
\end{tabular}

Для построения функции $\delta_{f r}=f(\tau)$ было выбрано пять уравнений, описывающих теплопроводность инея, область применения которых лежит в приемлемом диапазоне значений, а также две зависимости изменения плотности инея. Для графиков на рисунках 3-8 кривая 1 построена по данным эксперимента Бакулина, группа точек 2 является результатом расчета модели, где в качестве уравнения определения теплопроводности инея используется зависимость Шропа и Шмидта. Группы точек 3, 4 и 5 получены путем подстановки в модель зависимостей Ломакина и Чепурного, O'Neal, Tree и Lotz H., соответственно. Графики на рисунках 3, 5, 6, 7 - построены при использовании в модели зависимости предложенной Н.В. Ломакиным [20]. Для оценки влияния исходного уравнения плотности инея на кривую функции $\delta_{f r}=f(\tau)$, графики на рисунках 4,6 были построены на основании подстановки в модель уравнения Hayashi Y.

Для рисунках 3,5 , где в качестве уравнения плотности принято уравнение Н. В. Ломакина, кривые, по- строенные по уравнениям теплопроводности 2-5, дают малый разброс точек $\delta_{f r}=f(\tau)$. Следует отметить, что результаты, почти полностью совпадающие с экспериментальными данными, были получены при применении уравнения теплопроводности Н.В Ломакина и М.Н. Чепурного. На рисунках 4, 6, где в качестве уравнения плотности принято уравнение Hayashi Y., кривые функции $\delta_{f r}=f(\tau)$ характеризуются большим разбросом точек для используемых различных уравнений теплопроводности инея. В частности график на рисунке 4 показывает наибольшее расхождение, что связано с выходом режима № 44 из области применения уравнения Hayashi Y.

Проведенные расчеты зависимости $\delta_{f r}=f(\tau)$ по глубине пучка представлены на рисунках 7, 8. Для обоих рисунков в качестве уравнения плотности инея применялось уравнение Н. В. Ломакина. Задавшись толщиной инея отмеченной Бакулиным А.С. на первом ряду по истечении 12 часов работы воздухоохладителя получены кривые распределения толщины инея по глубине 
пучка. Максимальная погрешность по времени при расчете двух режимов составила $14 \%$, средняя $-5,5 \%$. Для всех уравнений теплопроводности инея модель дает малый разброс данных и сохраняет общую динамику изменения толщины инея по рядам. Режим № 19, где процесс выпадения инея происходит на более высоком температурном уровне, показал наилучшую сходимость с экспериментальными данными.

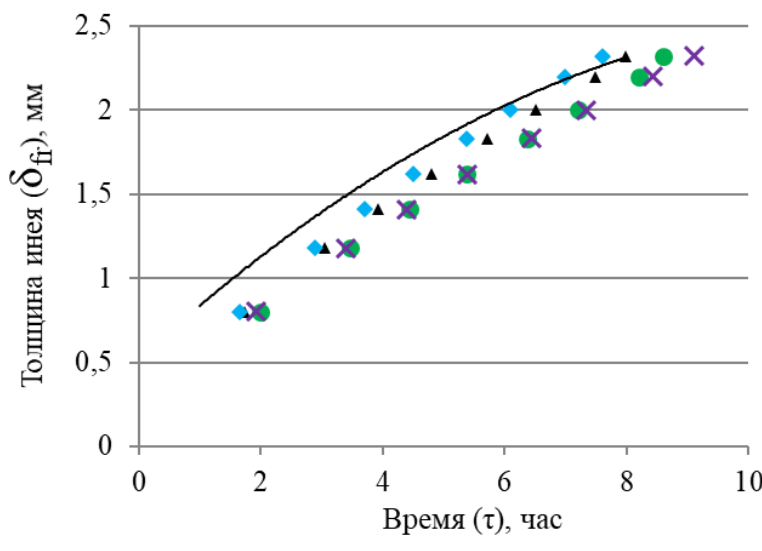

$1 \quad 0 \quad 2 \quad 3 \quad 4 \quad 4$

Рисунок 3. - Режим 44. Изменение толщины слоя инея на первом ряду. Уравнение плотности инея

Н.В. Ломакина.

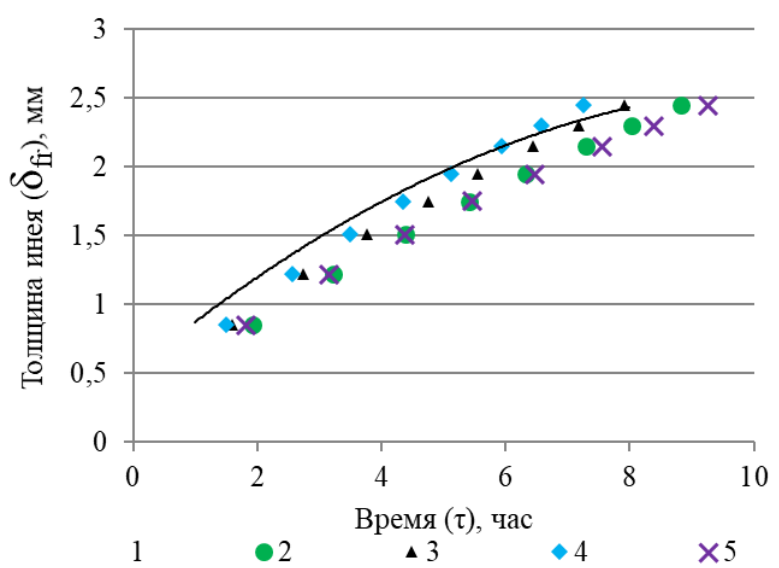

Рисунок 5. - Режим 42. Изменение толщины слоя инея на первом ряду. Уравнение плотности инея Н.В. Ломакина.

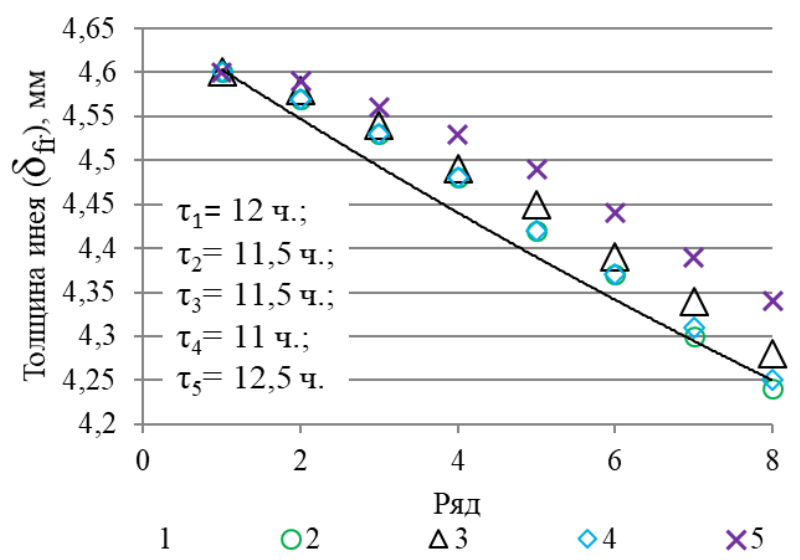

Рисунок 7. - Режим 19. Изменение толщины слоя инея по глубине пучка воздухоохладителя. Уравнение плотности инея Н.В. Ломакина.

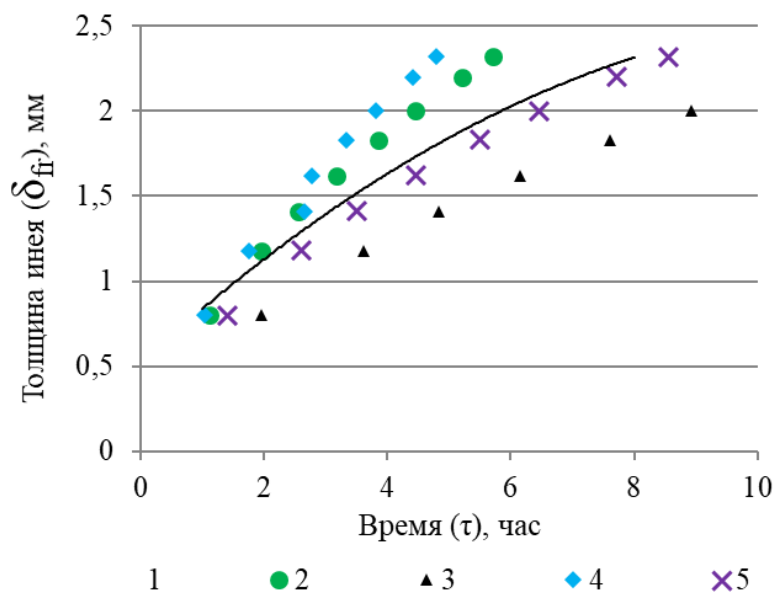

Рисунок 4. - Режим 44. Изменение толщины слоя инея на первом ряду. Уравнение плотности инея Hayashi Y.

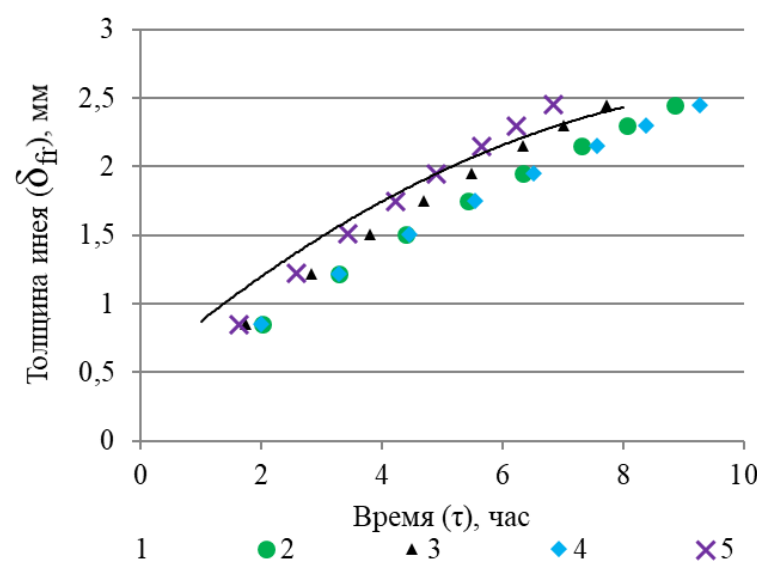

Рисунок 6. - Режим 42. Изменение толщины слоя инея на первом ряду. Уравнение плотности инея Hayashi Y.

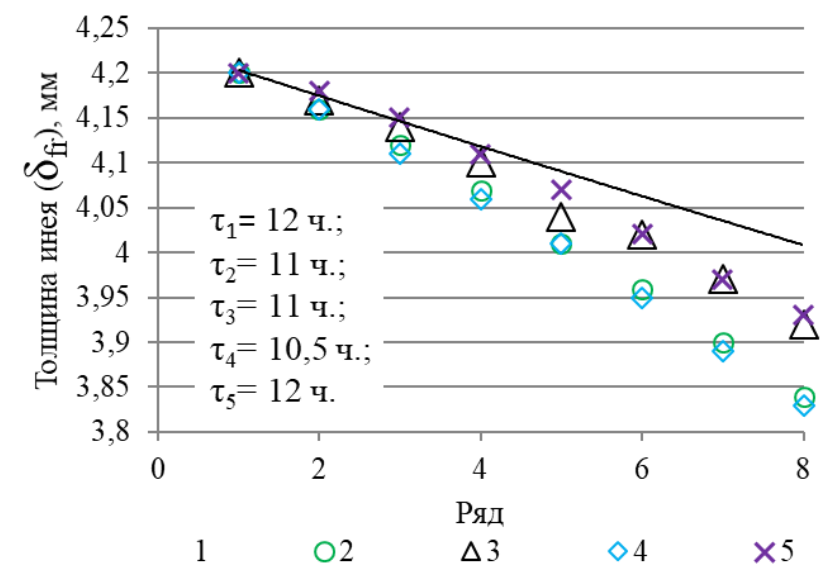

Рисунок 8. - Режим 39. Изменение толщины слоя инея по глубине пучка воздухоохладителя. Уравнение плотности инея Н.В. Ломакина. 


\section{Выводы}

В данной статье выполнен сравнительный расчет зависимостей $\delta_{f r}=f(\tau)$ при использовании различных уравнений плотности и теплопроводности инея. Сравнительный анализ показал, что уравнение, предложенное Н.В. Ломакиным является более универсальным с точки зрения диапазона возможных режимов воздухоохладителя. Вариантный расчет модели при различных исходных уравнениях плотности и теплопроводности инея показал, что комбинация зависимостей плотности и теплопроводности инея Ломакина-Чепурного [20], дают наилучшую сходимость с экспериментальными данными О.С. Бакулина для различных режимов как на первом ряду пучка, так и по его глубине. Также было отмечено, что применение различных уравнений плотности и теплопроводности инея а также их комбинаций, оказывают влияние на построение кривой $\delta_{f r}=f(\tau)$ иногда радикально, а следовательно, их применение должно быть обосновано. Предложенная математическая модель воздухоохладителя для всех уравнений зависимостей плотности и теплопроводности сохраняет тенденцию скорости роста инея, схожую с реальным экспериментом для всех режимов.

\section{Литература}

1. Напалков Г.Н. (1983). Тепломассоперенос в условиях инееобразования. - М.: Машиностроение. - с. 189 2. Lotz H. (1971) Wärmeund Stoffaustausch-vorgänge in bereifenden Lamellenrippen-Luftkühlern in Zusammenhang mit deren Betriebsverhalten. Kältetechnik-Klimatisierung, No 23, - P. 208-217

3. Бабакин Б.С., Еркин М.А. (1987) Интенсификация работы приборов охлаждения при инееобразовании: Обзорн. информ. -М.: АгроНИИТЭИММП. - 28 c.

4. Prins L. (1956) Wärme- und Stoffübertragung in einem querangeströmten, bereifenden Lüftkühler. -Kältetechnik, Bd, 8, Heft 6, P. 160-164.

5. Абдул Шакур Хан (1988) Инееобразования и тепломассообмен в профильных каналах прокатносварных воздухоохладителей //Дис. Канд.техн.наук Одесса. - 234 с.

6. J. D. Yonko, C. F. Sepsy. (1967) An Investigation of the Thermal Conductivity of Frost while Forming on a Flat Horizontal Plate," ASHRAE Transactions, Vol. 73, No. 2, P. 1.1-1.11.

7. O'Neal D.L., Tree D.R. (1985) A review of frost formation in simple geometries, ASHRAE Trans., Vol. 91. - P. 267-281.
8. Van Dusen M.S. (1929) International Critical Tables 5, McGraw-Hill Book Co., - P. 216

9. Ostin R., Anderson S. (1991) Frost growth parameters in a forced air stream, Int. J. of Heat Mass Transfer, Vol. 34 (4/5), - P. 1009-1017.

10. Sturm M., Holmgen J., Konig M., Morris, K. (1997) The thermal conductivity of seasonal snow,1. Glaciology, Vol. 43, - P. 26-41.

11. Lee K.S., Kim W.S., Lee T.H. (1997). A one dimensional model for frost formation on a cold flat surface. Int. J. Heat and Mass Transfer, 40(18), - P. 4359-4365.

12. Kandula M. Frost Growth and densification in laminar flow over flat surfaces. Int. J. Heat and Mass Transfer, 54(15), - P. 3719-3731.

13. Kandula M. (2011) Correlation of Water Frost Porosity in Laminar Flow over Flat Surfaces (Running Head: Water Frost Porosity in Laminar Flow). ASRC Aerospace, John F. Kennedy Space Center, FL, USA. - P.39.

14. Kandula M. (2010) On the Effective Thermal Conductivity of Frost Considering Mass Diffusion and Eddy Convection. ASRC Aerospace, John F. Kennedy Space Center, FL, USA. - P. 38.

15. Hosoda T. Uzuhashi H. (1967) Effects of frost on the heat transfer coefficient, Hitashi Review, Vol. 16, No.6, P. 254-259.

16. Yang D.K., Lee K.S. (2004) Dimensionless correlations of frost properties on a cold plate, Int. J. Refrig. No 27. - P. 89-96

17. Hayashi Y., Aoki A., Adachi S., and Hori K. (1977) Study of frost properties correlating with frost formation types, ASME J. of Heat Transfer, Vol. 99, - P. 239-245.

18. Hermes C.J.L., Piucco R., Barbosa J.R. (2009). A study of frost growth and densification on flat surfaces, Experimental Thermal and Fluid Science, Vol. 33, - P. 371379.

19. Wang W., Guo Q.C., Lu W.P., Feng Y.C. (2012). A generalized simple model for predicting frost growth on cold flat plate, Int. J. Refrig. No 35. - P. 475-486

20. Ломакин В.Н., Чепурной М.Н. (1989) Исследование теплофизических свойств наморажи-ваемого инея. "Холодильная техника" №11, с. 32-35.

21. Лагутин А.Е., Козаченко И.С., Желиба Ю.А. (2017). Моделирование формирования инея на оребренной поверхности воздухоохладителя. Проб-лемы региональной энергетики. №2(34). - с. 64-71.

22. Бакулін О.С. Динаміка формування інею в повітроохолоджувачах камер зберігання молочної продукції (2000). Дисертація на здобуття наукового ступеня кандидата технічних наук. - Одеса. с. 253.

Отримана в редакції 12.07.2017, прийнята до друку 08.09.2017

\title{
Evaluation of influence of initial frost density and thermal conductivity on the results of the frozen layer formation speed predicting
}

\author{
I. S. Kozachenko ${ }^{\bowtie}$, A. E. Lagutin \\ Odessa National Academy of Food Technologies, 112 Kanatnaya str., Odessa, 65039, Ukraine \\ $\bowtie$ e-mail: ilya.s.kozachenko@gmail.com
}

The main dependences for determination of the density and thermal conductivity of frost are presented in the paper. The comparison of the most common dependences of thermal conductivity and density of frost is given. The results of calcu- 
lation of the mathematical model in the form of frost layer thickness growth charts on the first row in time, as well as the depth of the beam are presented. The comparative analysis of the most common dependences of the density and thermal conductivity of frost is carried out and the influence of these parameters on the final form of the function of thickness dependence on time is shown. The approbation of the model on available experimental data for four modes at various temperature and humidity levels was conducted. As a result of the work, a combination of the dependences of the frost density and thermal conductivity is determined, which allows to provide a low error of the mathematical model for a wide range of calculated modes.

Key words: Modeling; Air cooler; Frost formation; Frost heat conduction; Frost density; Heat and mass transfer.

\section{References}

1. Napalkov, G.N. (1983). Heat and mass transfer in conditions of frost formation [Teplomassoperenos $\mathrm{v}$ usloviyah ineeobrazovaniya]. Mashinostroenie, Moscow, 189 p. (in Russian)

2. Lotz, H. (1971) Wärmeund Stoffaustausch-vorgänge in bereifenden Lamellenrippen-Luftkühlern in Zusammenhang mit deren Betriebsverhalten. KältetechnikKlimatisierung, No 23, pp. 208-217

3. Babakin, B.S., Erkin, M.A. (1987) Intensification of cooling heat exchange equipment in conditions of frost formation [Intensifikatsiya rabotyi priborov ohlazhdeniya pri ineeobrazovanii]: AgroNIIT-EIMMP. 28 p. (in Russian) 4. Prins, L. (1956) Wärme- und Stoffübertragung in einem querangeströmten, bereifenden Lüftkühler. Kältetechnik, Bd, 8, Heft 6, pp. 160-164.

5. Abdul Shakur Han (1988) Frost formation and heat and mass transfer in channels of welded air cooler [Ineeobrazovaniya i teplomassoobmen v profilnyih kanalah prokatno-svarnyih vozduhoohladiteley. dis. kand. tehn. nauk] Odessa. 234 p.

6. Yonko, J.D., Sepsy, C.F.. (1967) An Investigation of the Thermal Conductivity of Frost while Forming on a Flat Horizontal Plate, ASHRAE Transactions, Vol. 73, No. 2, pp. 1.1-1.11.

7. O'Neal, D.L., Tree, D.R. (1985) A review of frost formation in simple geometries, ASHRAE Trans., Vol. 91. P. 267-281.

8. Van Dusen, M.S. (1929) International Critical Tables 5, McGraw-Hill Book Co., 216 p.

9. Ostin, R., Anderson, S. (1991) Frost growth parameters in a forced air stream, Int. J. of Heat Mass Transfer, Vol. $\quad 34 \quad(4 / 5), \quad-\quad$ P. $\quad 1009-1017$. https://doi.org/10.1016/0017-9310(91)90012-4

10. Sturm M., Holmgen J., Konig M., Morris, K. (1997) The thermal conductivity of seasonal snow, 1. Glaciology, Vol. 43, pp. 26-41. https://doi.org/10.1017/s0022143000002781

11. Lee, K.S., Kim, W.S., Lee, T.H. (1997). A one dimensional model for frost formation on a cold flat surface. Int. J. Heat and Mass Transfer, 40(18), - P. 4359-4365. https://doi.org/10.1016/s0017-9310(97)00074-4

12. Kandula, M. (2011) Frost Growth and densification in laminar flow over flat surfaces. Int. J. Heat and Mass Transfer, 54(15), - P. 3719-3731.

https://doi.org/10.1016/j.ijheatmasstransfer.2011.02.056
13. Kandula, M. (2011) Correlation of Water Frost Porosity in Laminar Flow over Flat Surfaces (Running Head: Water Frost Porosity in Laminar Flow). ASRC Aerospace, John F. Kennedy Space Center, FL, USA. pp. 79-87 https://doi.org/10.1615/specialtopicsrevporousmedia.v3.i1.7 0

14. Kandula, M. (2010) On the Effective Thermal Conductivity of Frost Considering Mass Diffusion and Eddy Convection. ASRC Aerospace, John F. Kennedy Space Center, FL, USA. 321-336 https://doi.org/10.1615/specialtopicsrevporousmedia.v1.i4.4 0

15. Hosoda, T. Uzuhashi, H. (1967) Effects of frost on the heat transfer coefficient, Hitashi Review, Vol. 16, No.6, pp. 254-259.

16. Yang, D.K., Lee, K.S. (2004) Dimensionless correlations of frost properties on a cold plate, Int. J. Refrig. No 27. pp. 89-96. https://doi.org/10.1016/s01407007(03)00118-x

17. Hayashi, Y., Aoki, A., Adachi, S., and Hori, K. (1977) Study of frost properties correlating with frost formation types, ASME J. of Heat Transfer, Vol. 99, pp. 239245. https://doi.org/10.1115/1.3450675

18. Hermes, C.J.L., Piucco, R., Barbosa, J.R. (2009). A study of frost growth and densification on flat surfaces. Experimental Thermal and Fluid Science, Vol. 33, pp. 371379. https://doi.org/10.1016/j.expthermflusci.2008.10.006

19. Wang, W., Guo, Q.C., Lu, W.P., Feng, Y.C. (2012). A generalized simple model for predicting frost growth on cold flat plate. Int. J. Refrig. No 35.pp. 475-486. https://doi.org/10.1016/j.ijrefrig.2011.10.011

20. Lomakin, V.N., Chepurnoy, M.N. (1989) Issledovanie teplofizicheskih svoystv namorazhi-vaemogo ineya. "Holodilnaya tehnika" №11, P. 32-35.

21. Lagutin, A.E., Kozachenko, I.S., Zheliba, Yu.A. (2017). Modeling of Frost Formation on Fin-and-Tube Surface of Air Cooler. Problemyi regionalnoy energetiki. №2(34). pp. 64-71. (in Russian)

22. Bakulin, O.S., (2000) Dynamic of frost formation on air coolers of milk storage cold chambers [Dinamika formuvannya ineyu $\mathrm{v}$ povitrooholodzhuvachah kamer zberigannya molochnoi produktsii dis. kand. tehn. nauk]. Odesa.253 p. (in Russian)

Received 12 July 2017 Approved 08 September 2017 Available in Internet 30 October 2017 